\title{
Research on the Potential Mechanism of
}

\section{Gentiopicroside Against Gastric Cancer Based on Network Pharmacology}

This article was published in the following Dove Press journal:

Drug Design, Development and Therapy

\author{
Yanxia Huang ${ }^{1,2, *}$ \\ Jiatong $\operatorname{Lin}^{1,3, *}$ \\ Weimin $\mathrm{Yi}^{1,2, *}$ \\ Qinghua Liu ${ }^{1,4}$ \\ Linhui Cao ${ }^{1,2}$ \\ Yongcong Yan ${ }^{1,4}$ \\ Anqi Fu (iD) ${ }^{5}$ \\ Tingxuan Huang ${ }^{5}$ \\ Yingcheng Lyu',3 \\ Qihui Huang ${ }^{1,2}$ \\ Jie Wang $\mathbb{B}^{1,4}$
}

'Guangdong Provincial Key Laboratory of Malignant Tumor Epigenetics and Gene Regulation, Sun Yat-sen Memorial Hospital, Sun Yat-sen University, Guangzhou, People's Republic of China; ${ }^{2}$ Department of Traditional Chinese Medicine, Sun Yat-Sen Memorial Hospital, Sun Yat-Sen University, Guangzhou, People's Republic of China; ${ }^{3}$ Department of Thoracic Surgery, Sun Yat-sen Memorial Hospital, Sun Yat-sen University, Guangzhou, People's Republic of China; ${ }^{4}$ Department of Hepatobiliary Surgery, Sun Yat-Sen Memorial Hospital, Sun Yat-Sen University, Guangzhou, People's Republic of China; ${ }^{5}$ Sun Yat-Sen University Zhongshan School of Medicine, Guangzhou, People's Republic of China

*These authors contributed equally to this work

Correspondence: jie Wang

Department of Hepatobiliary Surgery, Sun YatSen Memorial Hospital, Sun Yat-Sen University, Yanjiang West Road I07\#, Guangzhou 510I20, People's Republic of China

Email jiewsysu@163.com

Qihui Huang

Department of Traditional Chinese Medicine, Sun Yat-Sen Memorial Hospital, Sun Yat-Sen University, Yanjiang West Road 107\#,

Guangzhou 510120, People's Republic of China Email hqhui84I81833@sina.com
Background: Gastric cancer was still one of the commonly diagnosed cancer types and the third-most common cause of cancer-related death in the world. Gentiopicroside, which is extracted from the Gentianella acuta, is commonly used in both traditional treatment and modern clinical care; therefore, its anticancer effects have been attracted more attention. However, the systematic analysis of action mechanism of Gentiopicroside on gastric cancer (GC) has not yet been carried out.

Aim: A network pharmacology-based strategy combined with molecular docking studies and in vitro validation was employed to investigate potential targets and molecular mechanism of Gentiopicroside against GC.

Materials and Methods: Potential targets of Gentiopicroside, as well as related genes of GC, were acquired from public databases. Potential targets, and signaling pathways were determined through bioinformatic analysis, including protein-protein interaction (PPI), the Gene Ontology (GO), and the Kyoto Encyclopedia of Genes and Genomes (KEGG). Subsequently, molecular docking and cell experiments were performed to further verify the above findings.

Results: Our findings revealed that the anticancer activity of Gentiopicroside potentially involves 53 putative identified target genes. In addition, GO, KEGG, and network analyses revealed that these targets were associated with cell proliferation, metabolic process, and other physiological processes. Furthermore, we have proved that critical compound affected the expression of CCND1, CCNE1, p-AKT and p-P38 at protein levels. These findings provide an overview of the anticancer action of Gentiopicroside from a network perspective; meanwhile, it might also set an example for future studies of other materials used in traditional Chinese medicine (TCM).

Conclusion: This study comprehensively illuminated the potential targets and molecular mechanism of Gentiopicroside against GC. It also provided a promising approach to uncover the scientific basis and therapeutic mechanism of TCM treating for disease.

Keywords: Gentiopicroside, gastric cancer, mechanism, network pharmacology, molecular docking

\section{Introduction}

According to the estimates from the International Agency for Research on Cancer (IARC) GLOBOCAN project, gastric cancer was still the fifth-most commonly diagnosed cancer type in 2018 and the third-most common cause of cancer-related death. ${ }^{1}$ And it is a multifactorial disease concerning Helicobacter pylori infection, lifestyle, genetic, or other risk factors. ${ }^{2}$ Which has posed a formidable potential threat to public health owing to its high morbidity and mortality. ${ }^{3}$ As we all know, the 
therapies for GC have been well developed in recent decades, the treatment strategies for GC mainly include surgery, chemotherapy, radiotherapy, targeted therapies, and immunotherapy; nevertheless, its mortality remains relatively high as a result of frequent recurrence and metastasis. ${ }^{4}$ Traditional Chinese medicine mainly from natural products is one of the most abundant resources of active compounds for drug discovery, playing an important role in the history of disease treatments, such as artemisinin used to cure malaria, ${ }^{5}$ arsenic trioxide in the treatment of acute promyelocytic leukaemia, ${ }^{6,7}$ and berberine in the treatment of type 2 diabetes mellitus, ${ }^{8,9}$ et al. Besides, TCM has been widely used in anticancer therapeutic prescription for thousands of years in China, basing on multiple mechanisms of anticancer effects, including apoptosis induction, proliferation inhibition, metastasis suppression, multidrug resistance reversal and immune function regulation. ${ }^{10-12}$ The advantage of using TCM is related to its ability on affecting multiple signaling pathways and molecular targets cancerrelated, while causing few side effects. ${ }^{13}$ Gentiopicroside isolated from Gentianella acuta, which has been shown to exert a wide spectrum of pharmacological activities, such as ameliorates diabetic nephropathy, ${ }^{14}$ reducing Pain, Pruritus, and Corticosteroid Addictive Dermatitis, ${ }^{15}$ as well as antidepressant-like effects, ${ }^{16}$ and so on. Close attention has been paid to Gentiopicroside due to its potential role in the treatment of cancer. ${ }^{17-19}$ Accordingly, the molecular mechanisms which Gentiopicroside induces and the resulting changes in cellular phenotypes are widely studied. Meanwhile, employment of computational methodologies for the identification of drug targets and the underlying mechanisms is becoming mainstream for further study. In particular, computational target identification and following the molecular mechanisms can accelerate the drug discovery and drug design process. ${ }^{20-23}$

Therefore, we elucidated the pharmacological actions of Gentiopicroside on gastric cancer systematically using computational methodologies. Then, we used the molecular docking and a series of in vitro assays to validate its effects on GC. A schematic diagram of the analysis procedures for Gentiopicroside target gene prediction is shown in Figure 1.

\section{Materials and Methods}

\section{Computational Targets Fishing}

Structural information of Gentiopicroside (Figure 2A, CAS:20831-76-9) was obtained from the NCBI PubChem (https://pubchem.ncbi.nlm.nih.gov/). At the same time, the protein targets of this compound were retrieved both from the TCMSP (http://lsp.nwu.edu.cn/tcmsp.php) and Pharmmapper (http://www.lilab-ecust.cn/pharmmapper/). Data on the Gastric cancer-related targets were obtained from two databases: (i) We obtained 137 Gastric cancerrelated targets from the Online Mendelian Inheritance in $\operatorname{Man}^{24}$ (http://omim.org/), an information platform mainly designed for human genes and genetic diseases. (ii) We obtained 18,223 Gastric cancer-related targets from the GeneCards: The Human Gene Database ${ }^{25}$ (https://www. genecards.org/). Common targets for Gentiopicroside and GC were obtained using Venny 2.1 (http://bioinfogp.cnb. csic.es/tools/venny/index.html). Common targets for Gentiopicroside and GC were obtained using Venny 2.1 (http://bioinfogp.cnb.csic.es/tools/venny/index.html).

\section{Protein-Protein Interaction (PPI) Network Construction}

The common targets related to Gentiopicroside treatment of GC were imported into the STRING database (https:// string-db.org/) to obtain their interaction relationship, "Homo sapiens" was chosen and a scoring value $>0.9$ was selected as the high confidence basis for protein interactions. Then, the common targets PPI network files were imported into Cytoscape 3.6.0 to merge and got the intersection of PPI network and core genes.

\section{Gene Function and Pathway Enrichment Analysis}

Gene ontology (Go) terms and Kyoto Encyclopedia of Genes and Genomes (KEGG) pathway enrichment analyses were performed by WebGestalt (http://www.webges talt.org/option.php).

\section{Network Construction}

In order to understand the complex relationships among compound, targets, and diseases, we used Cytoscape ${ }^{26}$ (https://www.nigms.nih.gov/) to construct and analyze the networks. It provides a powerful set of data integration, analysis, and visualization functions to analyze the complicated networks.

\section{Molecular Docking Analysis}

Autodock vina 1.1.2 was used for network pharmacologybased prediction and analysis and illustrated the mechanism of ligand acting on a complex molecular network by applying high-precision docking simulation and molecular 


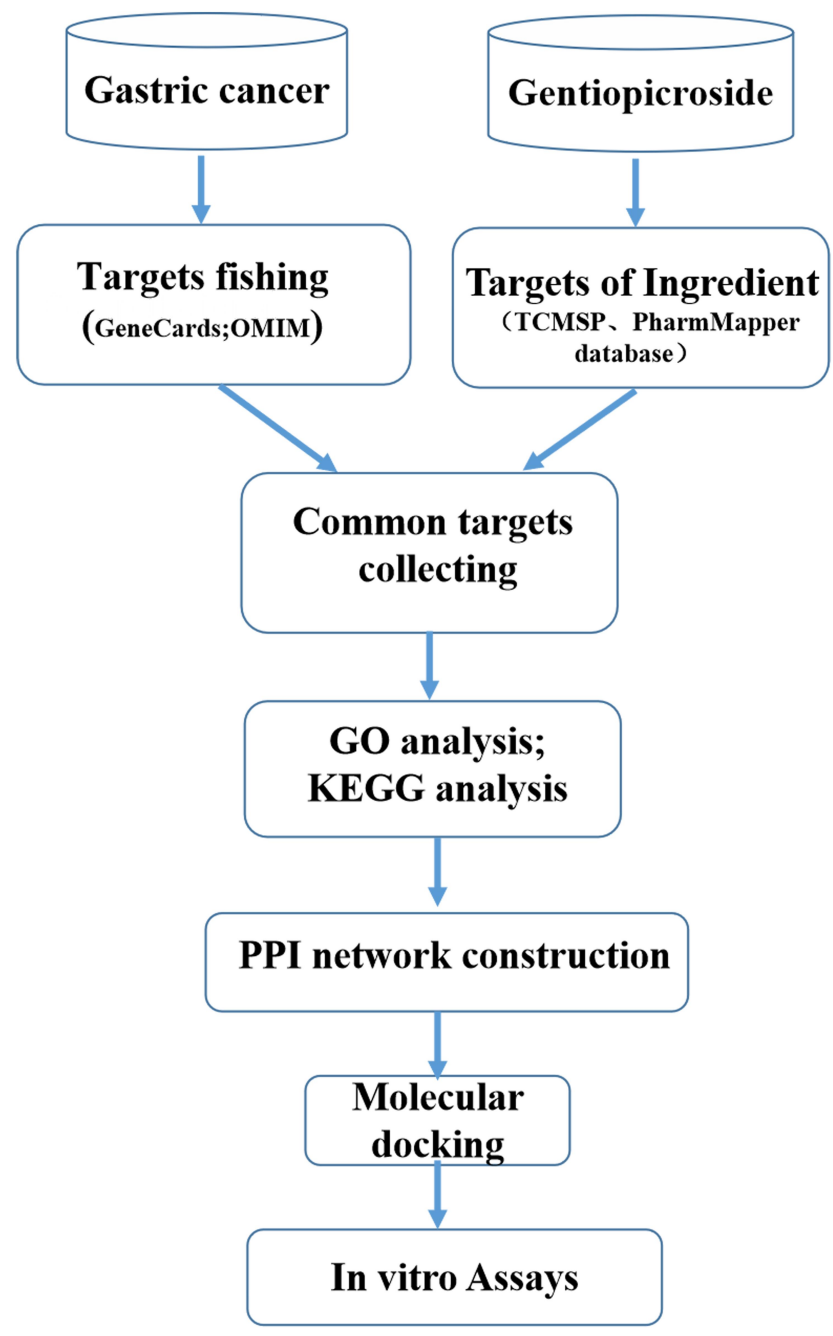

Figure I Workflow for the identification potential mechanism of Gentiopicroside in treating gastric cancer.

pathway map. We used Autodock vina to evaluate the binding potential between selected target and thecompound Gentiopicroside.

\section{Cell Culture}

The human gastric cancer cell lines HGC-27, MGC-803, BGC-823, AGS, and GES-1 were purchased from Chinese Academy of Sciences Shanghai Cell Bank and were cultured in Dulbecco's modified Eagle's medium supplemented with 10\% fetal bovine serum (Invitrogen, Carlsbad, CA, USA).

\section{Cell Proliferation Assay}

The cell viability of GC cells was measured by MTT assay, cells were plated into 96-well plates at a density of 2000 cells/well. After an overnight culture, cells were treated in DMEM with $0,25,50,100,200,400 \mu \mathrm{M}$ of Gentiopicroside for $48 \mathrm{hrs}$. Then, the cells were incubated with 3-(4, 5-dimethylthiazol-2-yl)-2, 5-diphenyltetrazolium bromide (MTT) for $4 \mathrm{~h}$ at $37^{\circ} \mathrm{C}$. The absorbance at $490 \mathrm{~nm}$ was measured.

\section{Flow Cytometry}

Cell cycle analysis was performed $48 \mathrm{~h}$ after transfection with propidium iodide staining as described previously. Cell apoptosis was also analyzed $48 \mathrm{~h}$ by Annexin $\mathrm{V}$ and propidium iodide staining as described previously. All the samples were assayed in triplicate.

\section{Western Blot Analysis}

Protein were separated by $10 \%$ sodium dodecyl sulfate-polyacrylamide gel electrophoresis (SDS-PAGE), and transferred to $0.22 \mu \mathrm{m}$ nitrocellulose membranes (Sigma) and incubated with specific primary antihuman antibodies. They were then incubated with specific antibodies according to the manufacturer's protocol. The GAPDH antibody was used as the control.
A

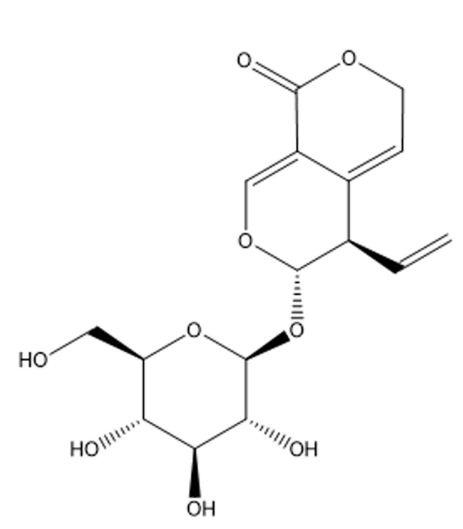

B

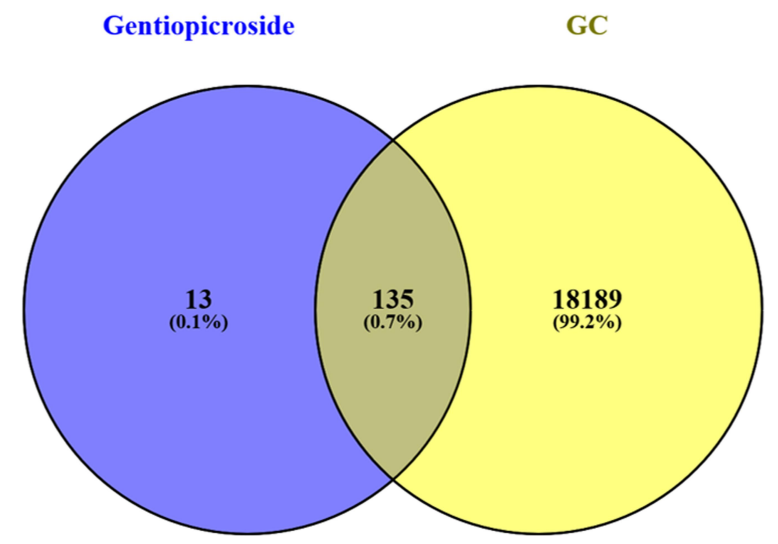

Figure 2 The venn diagram of the targets both in gastric cancer targets and Gentiopicroside targets. 


\section{Statistical Analysis}

Comparisons between groups were analyzed using the Student's t-test and one-way analysis of variance (ANOVA) by GraphPad Prism 7.0 (GraphPad Software Inc., California, USA). $\mathrm{P}<0.05$ was considered to indicate a statistically significant difference.

\section{Results}

\section{Targets Fishing}

There are a total of 148 drug-related targets were retrieved. Afterwards, from OMIM and GeneCards database, there were 18,324 gastric caner-related target genes. Then, we inputted drug targets and disease targets into Venny 2.1, after eliminating the redundancy we found 135 common targets between Gentiopicroside and $\mathrm{GC}$, the common targets are listed in Figure 2B.

\section{Target Protein Interaction Network Analysis}

The common targets were imported into the STRING database to obtain their interaction relationship, and a scoring value $>0.9$ was selected as the high confidence basis for protein interactions. The interaction network has 135 nodes and 64 edges (Figure 3A). TSV data were imported into Cytoscape, and the results showed a total of 61 nodes and 64 edges. Then, 10 targets were further screened as the center

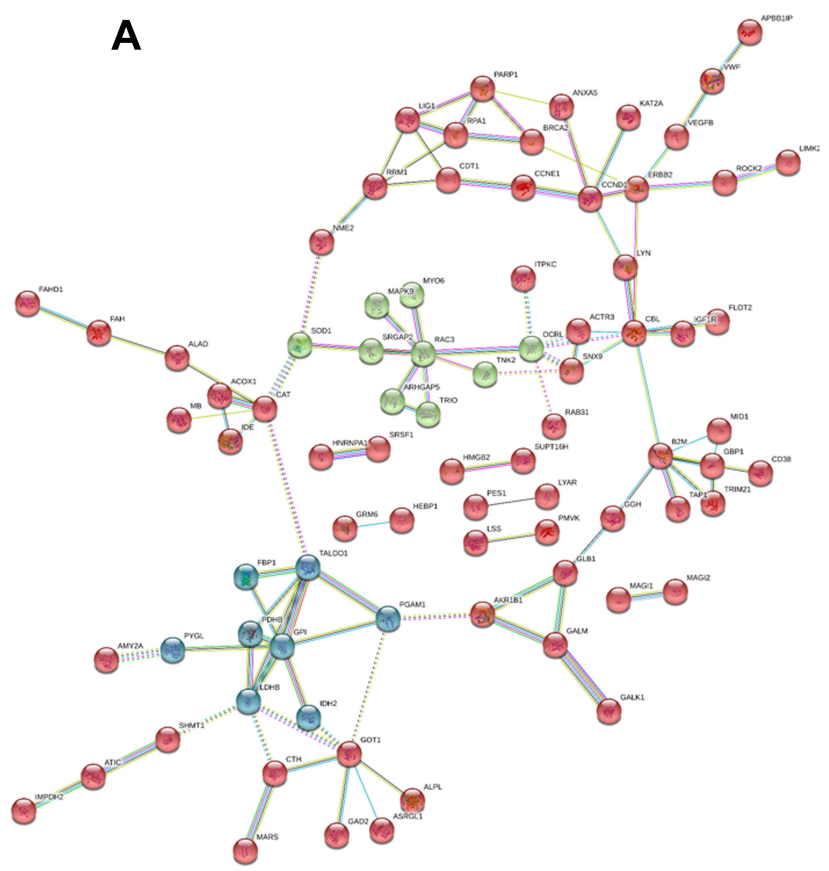

genes, including CAT, GOT1, LDHB, OCRL, CBL, CCND1, RAC3, GPI, B2M, TALDO1 (Figure 3B).

\section{GO and Pathway Analysis}

In order to obtain a more detailed understanding of the potential mechanisms of Gentiopicroside acting on gastric cancer, GO function and Pathway analysis were performed using WebGestalt. As shown in Figure 4A, the top seven functions were used as metabolic process, biological regulation, response to stimulus, cellular component, cell proliferation, reproduction, growth. These functional terms are highly relevant to anti-tumor activities. As for pathway analysis, the 53 targets participate in 10 KEGG pathways with significant $P$-value $<0.01$ including Metabolic pathways, PI3K-Akt signaling pathway and so on, which are shown in Figure 4B.

\section{Network Analysis}

Based on target and pathway analyses, the networks of the active compound and associated targets showed the relationships between compound and targets. As shown in Figure 5, this compound, disease, targets and pathways interaction network has 65 nodes and 168 edges. The red, blue, pink and green nodes represent the disease, Pathways, targets and compound; respectively, the edges represent the interactions among them.

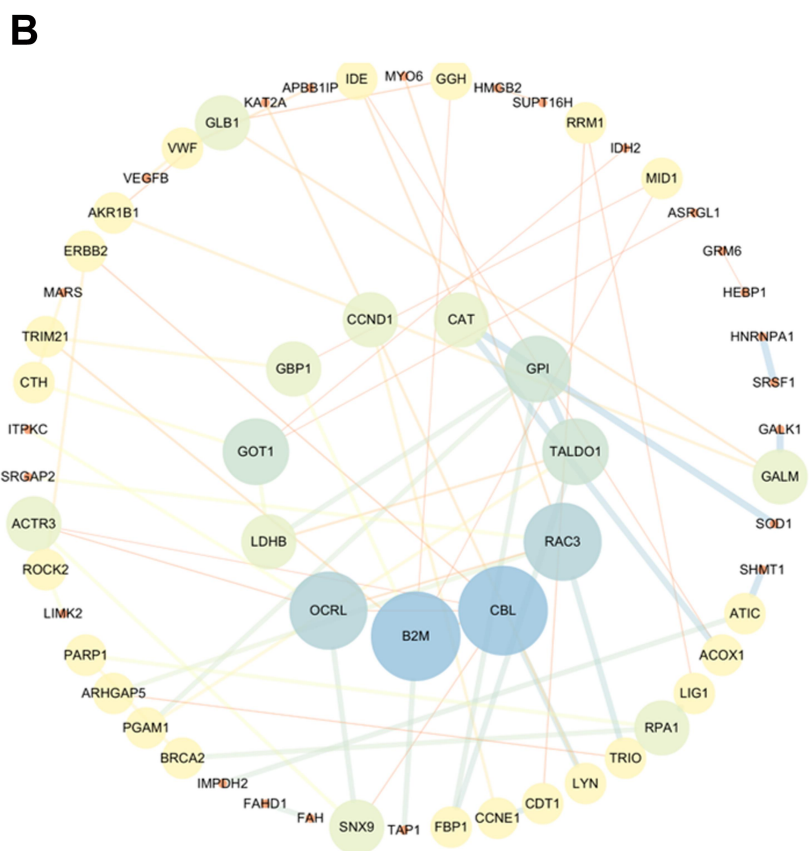

Figure 3 Construction of the protein-protein interaction network of Gentiopicroside and gastric cancer' associated targets. (A) Clusters of screened PPI networks. (B) The top 10 potential effective targets in gastric cancer. 
A
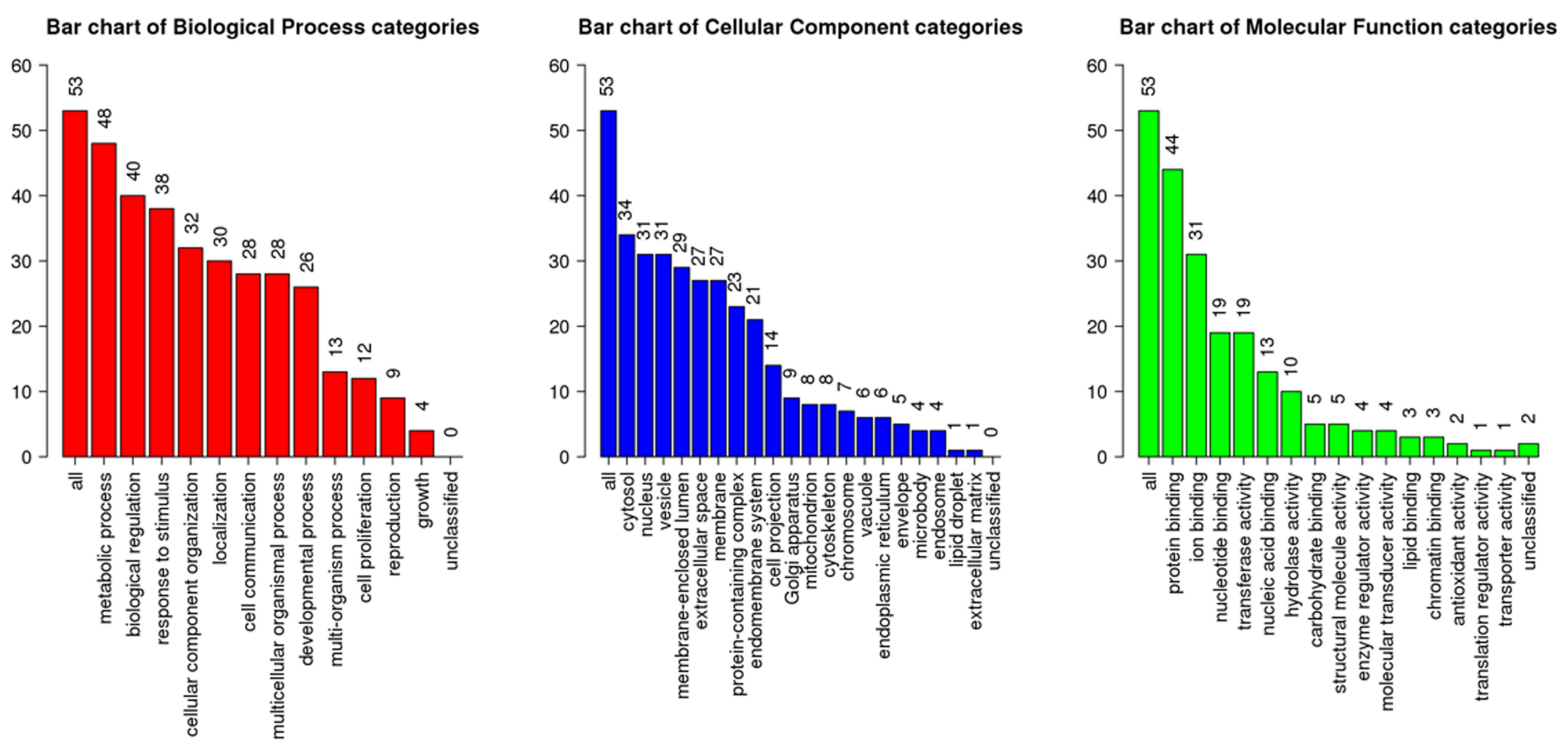

B

$\square \mathrm{FDR} \leq 0.05 \square \mathrm{FDR}>0.05$

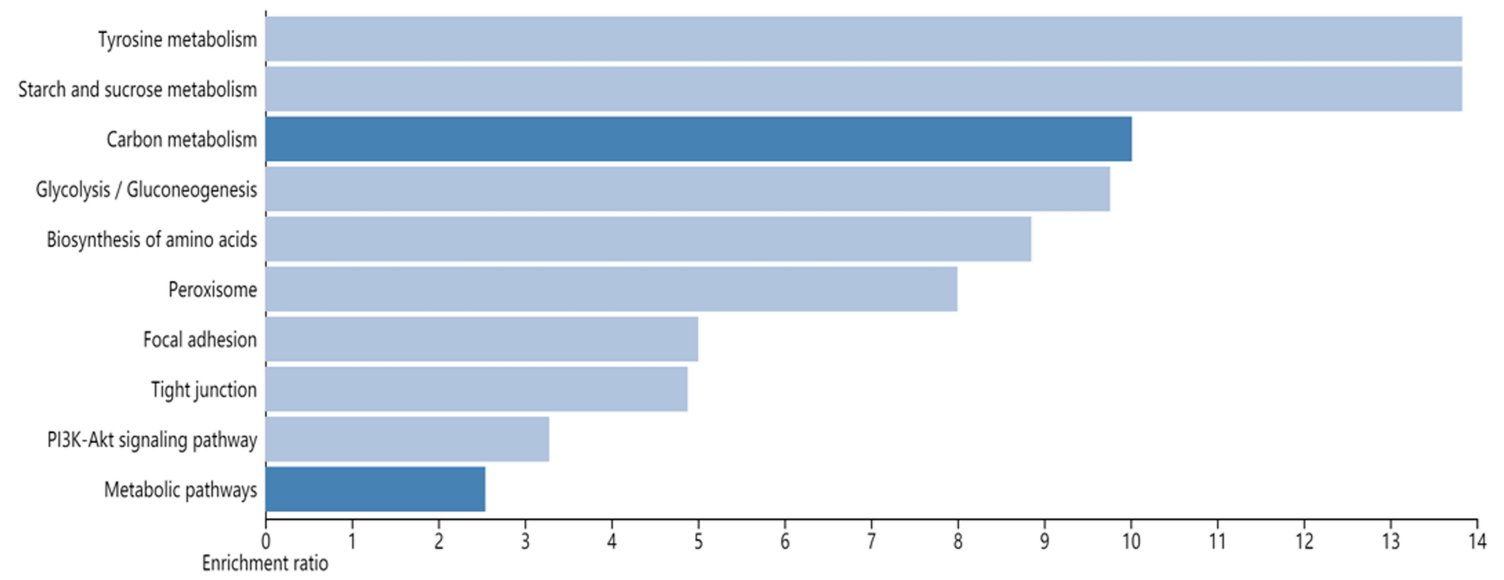

Figure $4 \mathrm{GO}$ analysis and KEGG analysis for the major targets of Gentiopicroside. (A) GO map of putative target genes. (B) KEGG pathway analysis of putative target genes.

\section{Docking Results Analysis}

We selected CCND1 as the target gene for molecular docking. This gene was mapped to the compound for molecular docking. Affinity was the score for the molecular docking, and when the score was lower, the binding affinity was stronger. An affinity $<-7$ indicated a stronger binding activity. ${ }^{27}$ The vina score of Gentiopicroside for treating gastric cancer was $-5.4 \mathrm{kcal} \mathrm{mol}^{-1}$. In Figure 6, we have shown the combination of the compound and gene in a 3D graph. The results indicated that Gentiopicroside had a good binding activity to CCND1.

\section{The Effects of Gentiopicroside on the Regulation of GC}

We assessed the effects of Gentiopicroside on GC cell viability. Four human GC cell lines and GES-1 were exposed to different concentrations of Gentiopicroside for 48hrs. As shown in Figure 7A, Gentiopicroside exerted obvious growth inhibition of $\mathrm{GC}$ cells with $\mathrm{IC}_{50}$ values of about $62-205 \mu \mathrm{M}$ for $48 \mathrm{hrs}$, of which HGC-27 was the most sensitive to the cytotoxic effect of Gentiopicroside ( $\mathrm{IC}_{50}$ value: $62.03 \pm 7.63 \mu \mathrm{M}$ ), whereas GES-1 was the least sensitive. The results indicate that at a certain dosage 


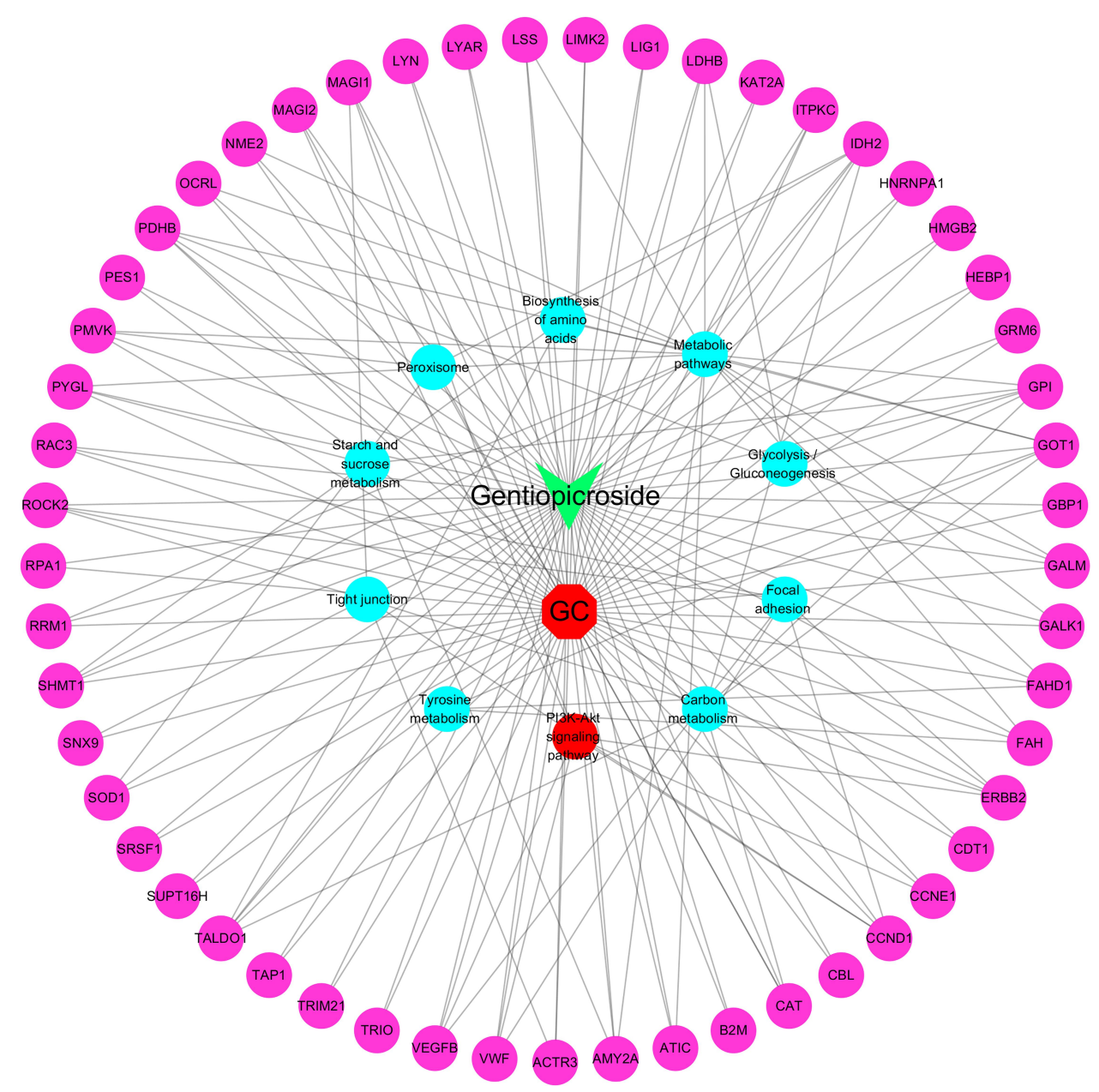

Figure 5 Drug-GC-targets-Pathways PPI network (The red, blue, pink and green nodes represent the disease, Pathways, targets and compound; respectively, the edges represent the interactions among them).

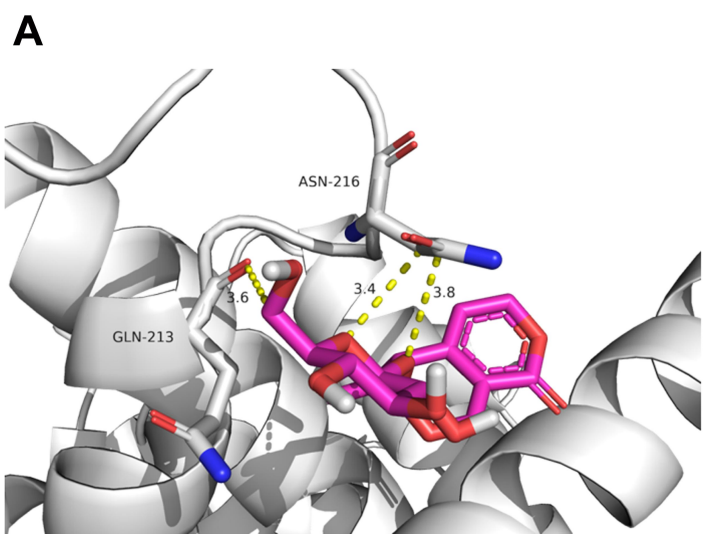

B

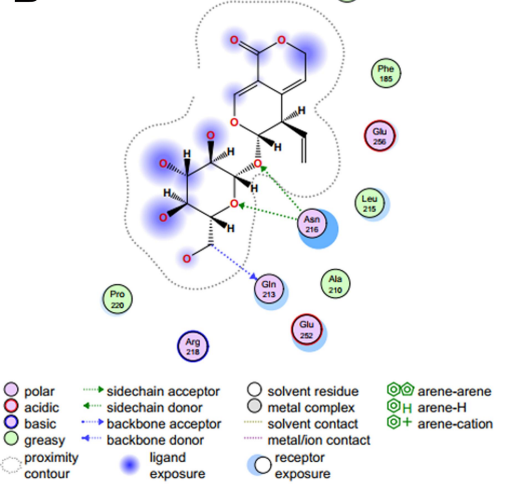

Figure 6 Molecular docking. (A) Stereogram of the interaction between CCNDI and Gentiopicroside. (B) Plane chart of the interaction between CCNDI and Gentiopicroside.

range, Gentiopicroside might selectively kill human GC cells rather than normal human gastric epithelial cells. And in the HGC-27 cell, the vitality of different concentration of Gentiopicroside was significantly inhibited compared with control groups (Figure 7B). Thus, we selected HGC-27 cell in the next assays. 
A

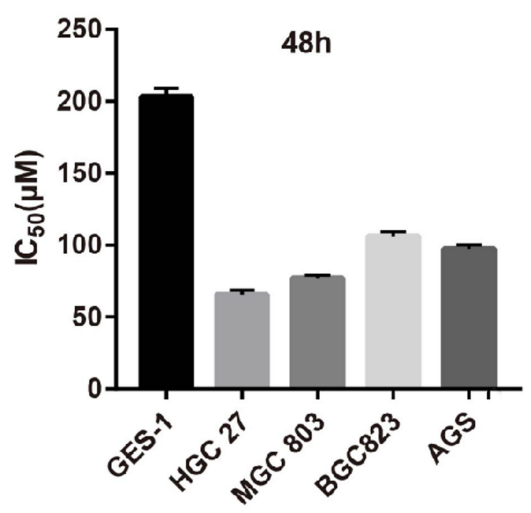

B

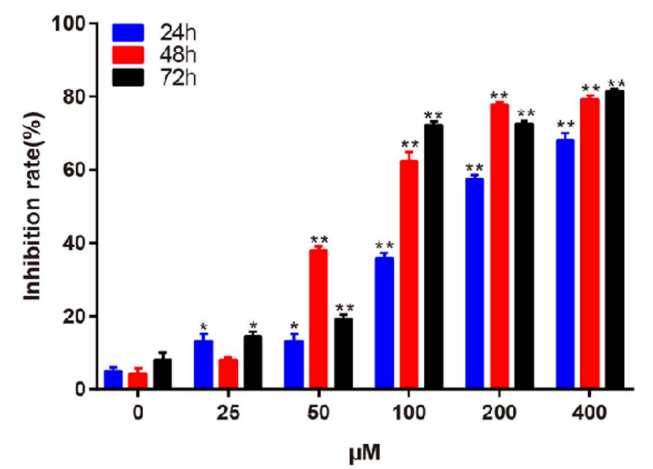

Figure 7 (A) Gentiopicroside displayed a significant growth inhibition in GC cells with IC50 values. (B) Gentiopicroside suppressed HGC-27 cells proliferation significantly under the concentration of $0,25,50,100,200,400 \mu \mathrm{M}$ with $24 \mathrm{~h}, 48 \mathrm{~h}$ and $72 \mathrm{~h}$ incubation, respectively. $\left({ }^{*} \mathrm{P}<0.05, * * \mathrm{P}<0.01\right)$.

\section{Gentiopicroside Induces Apoptosis and Cell Cycle Arrest}

Flow cytometry analysis was performed to evaluate the effects of Gentiopicroside on apoptosis and cell cycle distribution of HGC-27 cells. The results indicated that the proportion of these cells in apoptosis was significantly increased after treating by Gentiopicroside (Figure 8A). Cell cycle distribution showed that the proportion of HGC-27 cells was increased in the $\mathrm{G} 0 / \mathrm{G} 1$ phase, but decreased in the $\mathrm{S}$ phase in the Gentiopicroside-treated group (Figure 8B).

\section{Effects of Gentiopicroside on Potential}

\section{Targets in vitro}

In this study, we selected CCND1, CCNE1, AKT, P38 as the potential targets of Gentiopicroside combined with the basis of network pharmacology. Western blotting analysis
A

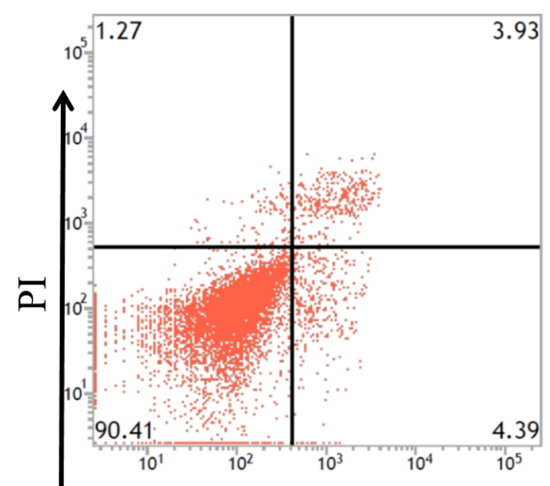

Annexin V FITC

B

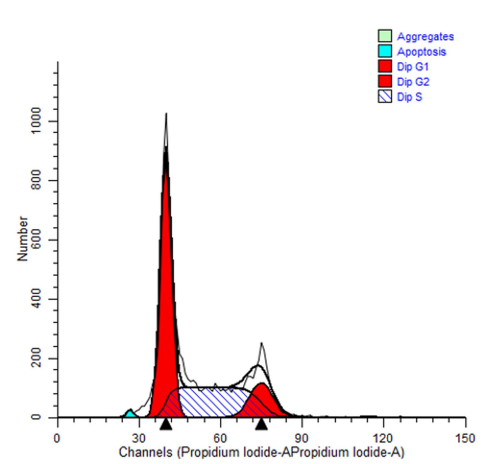

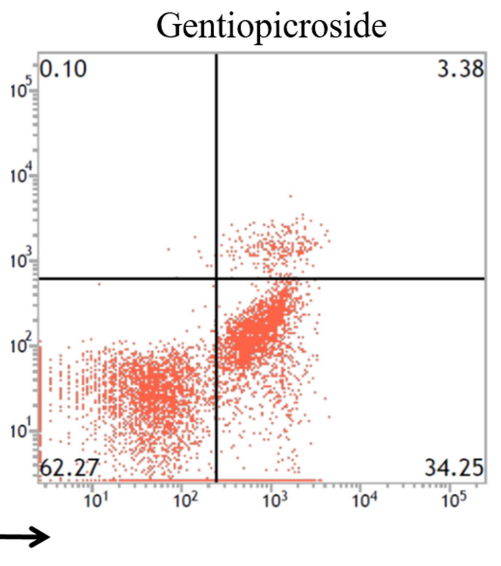

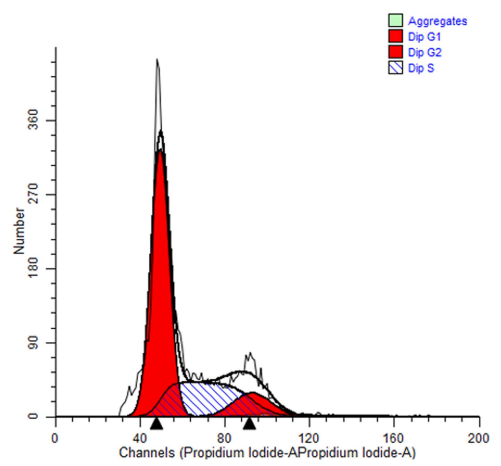

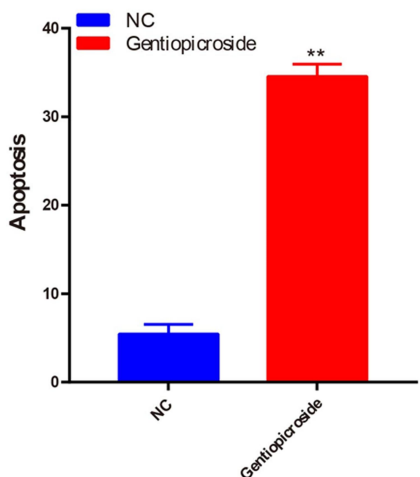

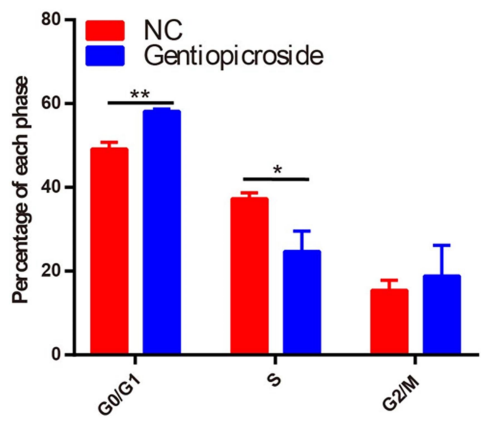

Figure 8 (A) Gentiopicroside suppressed HGC-27 cell apoptosis significantly under the concentration of $100 \mu$ M with 48 h incubation, respectively. (B) Gentiopicroside suppressed HGC-27 cell cycle significantly under the concentration of $100 \mu \mathrm{M}$ with $48 \mathrm{~h}$ incubation, respectively. $(* \mathrm{P}<0.05, * * \mathrm{P}<0.0 \mathrm{I})$. 
A

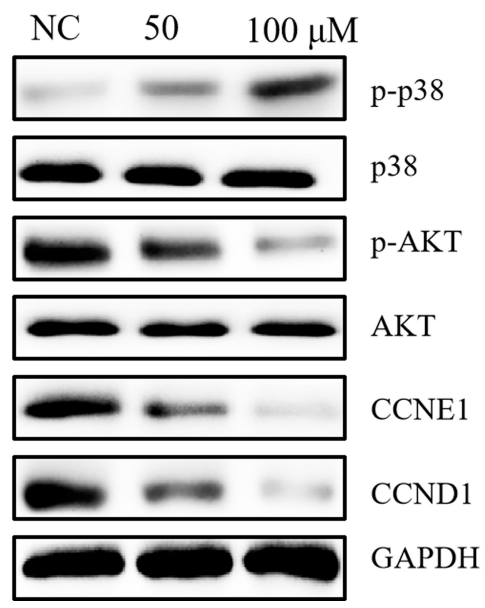

B

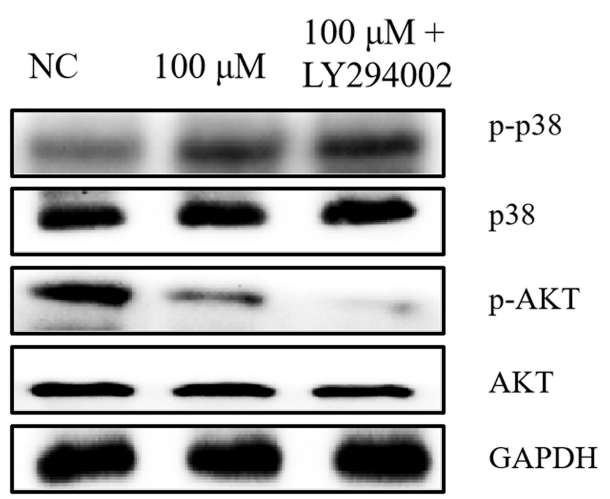

Figure 9 Effects of Gentiopicroside and LY294002 on the expression of PI3K/AKT pathway in HGC-27 cells. (A) WB confirmed that Gentiopicroside reduced CCNDI, CCNEI and P-AKT protein expression. While p-P38 significantly increased after stimulation with Gentiopicroside for 48 h. (B) HGC-27 cells were treated with Gentiopicroside and LY294002 as indicated, AKT, P38, p-P38 and p-AKT protein levels were determined using Western blotting.

was used to evaluate these protein levels in vitro. As shown in Figure 9, p-P38 protein levels were significantly increased in the Gentiopicroside treated group compared with the control group. However, the protein expression of p-AKT, CCND1, CCNE1 was significantly attenuated after Gentiopicroside treatment (Figure 9A). What is more, after using LY294002 to inhibit the PI3K/AKT pathway, the level of p-AKT inhibited further, and it also has a significant effect on p-P38 (Figure 9B).

\section{Discussion}

Traditional Chinese medicine (TCM) has more than 2000 years of history and has gained widespread clinical applications. However, the explicit role of TCM in preventing and treating $\mathrm{GC}$ remains unclear due to lack of sound scientific evidence. Mostly it is very hard to clarify the effective mechanism of TCM because of its multiple ingredients and targets. In recent years, virtual pharmacology research was regarded as the fastest and most effective screening method in the early study of drug effectiveness. $^{21,23,28-32}$ Gentianella acuta is an old drug commonly used for treating hepatitis and carcinoma. Therefore, in this study, Gentiopicroside was employed to study the gastric cancer enriching mechanism by network pharmacology approach. We integrated information from publicly available databases to predict interactions between Gentiopicroside and its potential targets in GC, as well as the signaling pathways and networks involved. In this study, 53 targets were screened and participated in 10 pathways based on P-values of each enriched pathway and their relationship to GC, KEGG analysis of the core PPI network indicated that Gentiopicroside may result in multiple effects for gastric cancer treatment by regulating multiple pathways. Furthermore, CCND1 with higher score, which was closely related to the occurrence and development of GC, was selected for molecular docking simulation by the LeDock. And the possible mechanism of Gentiopicroside inhibited tumor development through regulating PI3K-Akt signaling pathway. Accumulating evidence indicates that the PI3K/AKT signaling pathway, which is one of the most frequently activated signaltransduction pathways in cancer, is involved in cell cycle, cell proliferation, growth, migration, angiogenesis, and apoptosis. These results are consistent with several studies in vitro suggesting that Gentiopicroside exhibited anticancer effects on ovarian cancer cell lines through induction of apoptosis, ${ }^{17}$ while inhibited Cell Growth and Migration on Cervical Cancer via the Reciprocal MAPK/ Akt Signaling Pathways. ${ }^{33}$ And to the best of our knowledge, this is the first study to report that Gentiopicroside exhibited anticancer effect on gastric cancer.

This study reports multicomponent therapeutics by Gentiopicroside to provide multitarget, multipathway regulation of GC activity. Although all of the findings in this paper gave evidence to prove the major active compound in Gentianella acuta to gastric cancer, the limitation of our study is that the molecular mechanism of this study was too simple, only a series of simple assays in vitro were 
carried. In future, more experiments are needed to provide support for our findings, and future studies should focus on providing experimental evidence and expanding the role of Gentianella acuta on gastric cancer.

\section{Acknowledgments}

This work is supported by the Guangdong Basic and Applied Basic Research Foundation (Grant No. 2019A1515111105) and the China Postdoctoral Science Foundation (Grant No. 2019M663323).

\section{Author Contributions}

Yanxia Huang, Jiatong Lin and Weimin Yi contributed equally. And all authors contributed to data analysis, drafting or revising the article, have agreed on the journal to which the article will be submitted, gave final approval of the version to be published, and agree to be accountable for all aspects of the work.

\section{Disclosure}

The authors report no conflicts of interest in this work.

\section{References}

1. Bray F, Ferlay J, Soerjomataram I, Siegel RL, Torre LA. Global cancer statistics 2018: GLOBOCAN estimates of incidence and mortality worldwide for 36 cancers in 185 countries. CA Cancer J Clin. 2018;68(6):394-424.

2. Alessio Biagioni LM, Skalamera I, Papucci L. Update on gastric cancer treatments and gene therapies. Cancer Metastasis Rev. 2019. doi:10.1007/s10555-019-09803-7

3. Li SS, Costantino CL, Mullen JT. Morbidity and Mortality of Total Gastrectomy: a Comprehensive Analysis of 90-Day Outcomes. $J$ Gastrointest Surg. 2019;23(7):1340-1348.

4. Thrift AP, El-Serag HB. Burden of Gastric Cancer. Clin Gastroenterol Hepatol. 2019;2:124.

5. Tu Y. The discovery of artemisinin (qinghaosu) and gifts from Chinese medicine. Nat Med. 2011;17(10):1217-1220.

6. Emadi A, Gore SD. Arsenic trioxide - an old drug rediscovered Blood Rev. 2010;24(4-5):191-199.

7. Wang ZY, Chen Z. Acute promyelocytic leukemia: from highly fatal to highly curable. Blood. 2008;111(5):2505-2515.

8. Li WL, Zheng HC, Bukuru J, De Kimpe N. Natural medicines used in the traditional Chinese medical system for therapy of diabetes mellitus. J Ethnopharmacol. 2004;92(1):1-21.

9. Wang J, Wong YK, Liao F. What has traditional Chinese medicine delivered for modern medicine? Expert Rev Mol Med. 2018;20:e4.

10. Schmeda-Hirschmann G, Quispe C, Arana GV, Theoduloz C, Urra FA, Cárdenas C. Antiproliferative activity and chemical composition of the venom from the Amazonian toad Rhinella marina (Anura: bufonidae). Toxicon. 2016;121::119-129. doi:10.1016/j. toxicon.2016.09.004

11. Ma L, Wang B, Long Y, Li H. Effect of traditional Chinese medicine combined with Western therapy on primary hepatic carcinoma: a systematic review with meta-analysis. Front Med. 2017;11 (2):191-202. doi:10.1007/s11684-017-0512-0
12. Chon S-H, Berlth F, Plum PS, et al. Gastric cancer treatment in the world: germany. Translational Gastroenterology Hepatol. 2017;2 (5):53. doi:10.21037/tgh.2017.05.07

13. Yan Z, Lai Z, Lin J. Anticancer Properties of Traditional Chinese Medicine. Comb Chem High Throughput Screen. 2017;20 (5):423-429. doi:10.2174/1386207320666170116141818

14. Xiao H, Sun X, Liu R, et al. Gentiopicroside activates the bile acid receptor Gpbar1 (TGR5) to repress NF-kappaB pathway and ameliorate diabetic nephropathy. Pharmacol Res. 2020;151: 104559 .

15. Chaoqun Zhou WD, Xiang G, Lin X. Gentiopicroside Reduces Pain, Pruritus, and Corticosteroid Addictive Dermatitis. Nat Prod Commun. 2019;14(9):1-4.

16. Yao T, Cui Q, Liu Z, Wang C, Zhang Q, Wang G. Metabolomic evidence for the therapeutic effect of Gentiopicroside in a corticosterone-induced model of depression. Biomed Pharmacother. 2019;120:109549.

17. Xiaohao L, Hao Shen CY. Gentiopicroside exerts convincing antitumor effects in human ovarian carcinoma cells (SKOV3) by inducing cell cycle arrest, mitochondrial mediated apoptosis and inhibition of cell migration. JBUON. 2019;24(1):280-284.

18. Xian-Ju Huang JL, Mei Z-Y. Gentiopicroside and sweroside from Veratrilla baillonii Franch induced phosphorylation of Akt and suppressed Pck1 expression in hepatoma cells. Biochem Cell Biol. 2016;94(3):270-278.

19. Chou CC. Pharmacological evaluation of several major ingredients of Chinese herbal medicines in human hepatoma Hep3B cells. Eur J Pharmaceutical Sci. 2003;1(19):403-412.

20. Shi XQ, Yue SJ, Tang YP, et al. A network pharmacology approach to investigate the blood enriching mechanism of Danggui buxue Decoction. J Ethnopharmacol. 2019;235::227-242.

21. Zhou C, Liu L, Zhuang J, et al. A Systems Biology-Based Approach to Uncovering Molecular Mechanisms Underlying Effects of Traditional Chinese Medicine Qingdai in Chronic Myelogenous Leukemia, Involving Integration of Network Pharmacology and Molecular Docking Technology. Med Sci Monit. 2018;24::4305-4316.

22. Wang T, Li HT, Wei SZ, et al. Use of Network Pharmacology and Molecular Docking to Investigate the Mechanism by Which Ginseng Ameliorates Hypoxia. Biomed Environ Sci. 2018;31 (11):855-858.

23. Gong B, Kao Y, Zhang C, Sun F. Systematic Investigation of Scutellariae Barbatae Herba for Treating Hepatocellular Carcinoma Based on Network Pharmacology. Evid Based Complement Alternat Med. 2018;2018:4365739.

24. Hamosh A, Scott AF, Amberger JS, Bocchini CA. Online Mendelian Inheritance in Man (OMIM), a knowledgebase of human genes and genetic disorders. Nucleic Acids Res. 2005;33:D514-D517.

25. Stelzer G, Rosen N, Plaschkes I, et al. The GeneCards Suite: from Gene Data Mining to Disease Genome Sequence Analyses. Curr Protoc Bioinformatics. 2016;54:30.

26. Shannon P, Markiel A, Ozier O, et al. Cytoscape: a software environment for integrated models of biomolecular interaction networks. Genome Res. 2003;13(11):2498-2504.

27. Trott O. AutoDock Vina: improving the speed and accuracy of docking with a new scoring function, efficient optimization, and multithreading. J Comput Chem. 2010;31(2):455-461.

28. Liu X, Wu J, Zhang D, Wang K, Duan X. A Network Pharmacology Approach to Uncover the Multiple Mechanisms of Hedyotis diffusa Willd. on Colorectal Cancer. Evid Based Complement Alternat Med. 2018;2018:6517034.

29. Zhang YF, Huang Y, Ni YH. Systematic elucidation of the mechanism of geraniol via network pharmacology. Drug Des Devel Ther. 2019;13::1069-1075.

30. Yu G, Wang W, Wang X. Network pharmacology-based strategy to investigate pharmacological mechanisms of Zuojinwan for treatment of gastritis. BMC Complement Altern Med. 2018;18(1):292. 
31. Bi YH, Zhang LH, Chen SJ. Antitumor Mechanisms of Curcumae Rhizoma Based on Network Pharmacology. Evid Based Complement Alternat Med. 2018;2018:4509892.

32. Meng Z, Liu X, Wu J, et al. Mechanisms of Compound Kushen Injection for the Treatment of Lung Cancer Based on Network Pharmacology. Evid Based Complement Alternat Med. 2019;2019:4637839.
33. Hu ZQ, Wang H, Fu Y, Ma K, Ma XY. Gentiopicroside Inhibits Cell Growth and Migration on Cervical Cancer via the Reciprocal MAPK/ Akt Signaling Pathways. Nutrition and Cancer. 2020;1-12. doi: $10.1080 / 01635581.2020 .1801777$

\section{Publish your work in this journal}

Drug Design, Development and Therapy is an international, peerreviewed open-access journal that spans the spectrum of drug design and development through to clinical applications. Clinical outcomes, patient safety, and programs for the development and effective, safe, and sustained use of medicines are a feature of the journal, which has also been accepted for indexing on PubMed Central. The manuscript management system is completely online and includes a very quick and fair peer-review system, which is all easy to use. Visit http://www. dovepress.com/testimonials.php to read real quotes from published authors. 